\title{
Chemiluminescence Detection of Five-membered Heteroaromatic Compounds Using Electrogenerated Tris(2,2'-bipyridine)- ruthenium(III)
}

\author{
Hitoshi Kodamatani,* Yu Komatsu,* Shigeo YamaZaKI,** and Keiitsu Saito**** \\ *Department of Environmental Chemistry, Kanazawa Institute of Technology, \\ 7-1 Ohgigaoka, Nonoichi, Ishikawa 921-8501, Japan \\ **Department of Applied Science, Okayama University of Science, 1-1 Ridaicho, Okayama 700-0005, Japan \\ ***Division of Natural Environment and Chemistry, Faculty of Human Development, Kobe University, \\ 3-11 Tsurukabuto, Nada, Kobe 657-8501, Japan
}

\begin{abstract}
Heteroaromatic compounds, such as 2,5-dimethylthiophene, 2,5-dimethylpyrrole, and 2,5-dimethylfuran, were found to act as reducing agents for the tris $\left(2,2^{\prime}\right.$-bipyridine)ruthenium(III), $\mathrm{Ru}(\mathrm{bpy}) 3_{3}{ }^{3+}$, chemiluminescent reaction. In order to characterize the chemiluminescent reaction of $\mathrm{Ru}(\mathrm{bpy}) 3^{3+}$ with heteroaromatic compounds, we have investigated various mono-, di-, and tri-heteroaromatic compounds. The $\pi$-electron density and stability of aromatic rings influence the chemiluminescent efficiency of the reaction. Above all, 2,5-dimethylthiophene produced strong chemiluminescence under acidic conditions. In addition, we confirmed that the rate of the chemiluminescent reaction of $\operatorname{Ru}(\mathrm{bpy})_{3}{ }^{3+}$ with $2,5-$ dimethylthiophene is very fast.
\end{abstract}

(Received January 25, 2007; Accepted March 1, 2007; Published April 10, 2007)

\section{Introduction}

Chemiluminescence $(\mathrm{CL})$ is a simple but good detection method for selected analytes in analytical chemistry. Tris $\left(2,2^{\prime}-\right.$ bipyridine)ruthenium(III), Ru(bpy) $3^{3+}$, has been shown to be an important CL reagent. ${ }^{1-6}$ In the $\mathrm{Ru}(\mathrm{bpy}) 3^{3+} \mathrm{CL}$ system, emission results from the relaxation of the excited state $\mathrm{Ru}(\mathrm{bpy})_{3}{ }^{2+*}$ to the ground state $\mathrm{Ru}(\mathrm{bpy})_{3}{ }^{2+}$. The excited state species is probably produced as follows. The suitable reducing agent is oxidized with $\mathrm{Ru}(\mathrm{bpy})_{3^{3+}}$ generating a strongly reducing radical intermediate. Additional $\mathrm{Ru}(\mathrm{bpy})_{3}{ }^{3+}$ reacts with this radical intermediate to form the excited state $\mathrm{Ru}(\mathrm{bpy})_{3}{ }^{2+*}$. Since Noffsinger and Danielson first reported the CL of Ru(bpy) $3^{3+}$ with aliphatic tertiary amines, ${ }^{7}$ a wide range of analytical applications have been reported..$^{8-16} \mathrm{Ru}(\mathrm{bpy})_{3}{ }^{3+}$ is used as a detection reagent for a variety of compounds having an amine moiety; various analytical methods are employed, such as highperformance liquid chromatography (HPLC), ${ }^{8-10}$ flow injection analysis (FIA), ${ }^{11,12}$ capillary electrophoresis, ${ }^{13,14}$ and micro-total analysis system. ${ }^{15}$ Since $\mathrm{Ru}(\mathrm{bpy})_{3}{ }^{3+}$ can be electrochemically

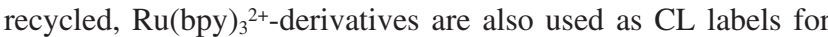
immunoassay and DNA assay with $\mathrm{Ru}(\mathrm{bpy})_{3}{ }^{2+} /$ tripropylamineelectrogenerated CL (ECL) system. ${ }^{16}$ The Ru(bpy) ${ }_{3}{ }^{2+}$ ECL-label can be sensitively determined at subpicomolar concentrations, over a wide dynamic range. These analytical applications are shown in a number of recent reviews ${ }^{1-5}$ and in a book. ${ }^{6}$ However, it is not clear what kinds of compound can become reducing agents for $\mathrm{Ru}(\mathrm{bpy}) 3^{3+} \mathrm{CL}$. Finding novel reducing

$\dagger$ To whom correspondence should be addressed.

E-mail: keisaito@kobe-u.ac.jp agents leads to extending this CL detection method to a wider range of compounds.

The authors recently showed that the aliphatic conjugated dienes cause $\mathrm{CL}$ upon mixing with $\mathrm{Ru}(\mathrm{bpy}) 3^{3+}$ in aqueous solutions and that the electron-withdrawing/donating character of the functional group attached to the carbon atoms building conjugated double bonds influences the chemiluminescent efficiency of the reaction. ${ }^{17}$ As additional experiments in the study, we have tested the chemiluminescent reaction of $\mathrm{Ru}$ (bpy) $3^{3+}$ with heteroaromatic compounds such as 2,5dimethylfuran (DMF), 2,5-dimethylpyrrole (DMP), and 2,5dimethylthiophene (DMT). Because DMF has some reactivity as an aliphatic conjugated diene, whereas DMT does not have such a property, we thought that DMF will give strong CL intensity and that DMT will not produce CL. However, contrary to our expectation, DMT gave the strongest CL intensity among these compounds. Moreover, we previously reported a HPLC method with $\mathrm{Ru}(\mathrm{bpy}) 3^{3+} \mathrm{CL}$ detection for determining 3-amino-1,2,4-triazole, which is one of the heteroaromatic compounds and is widely used as a herbicide. ${ }^{18}$ These findings led us to study the chemiluminescent reaction of $\mathrm{Ru}(\mathrm{bpy})_{3}{ }^{3+}$ with various heteroaromatic compounds. On the basis of the findings obtained, we have discussed the relationship between the structural attributes of heteroaromatic compounds and the observed CL intensities.

\section{Experimantal}

\section{Chemicals}

All chemicals were of reagent grade or better and were used without further purification, except for tris( $2,2^{\prime}$-bipyridine $)$ - 
(A)

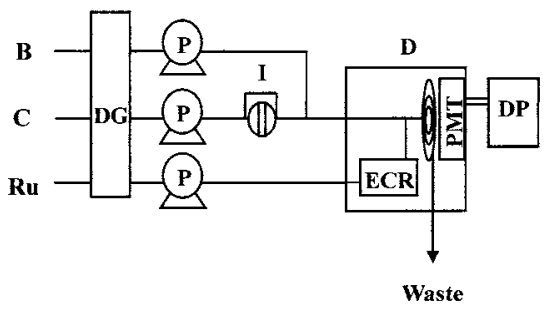

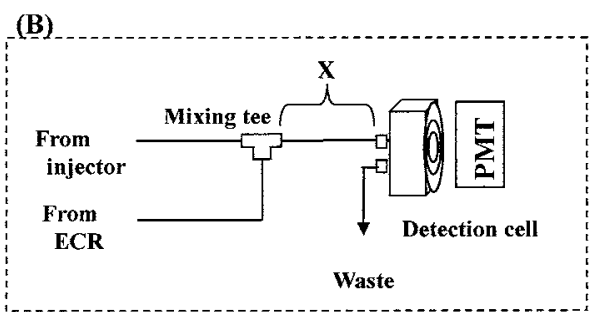

Fig. 1 (A) Schematic diagrams of the FIA-CL system and (B) an enlarged illustration of the detection cell. C, Carrier solution; Ru, Ru(bpy) $3^{3+}$ solution; B, buffer solution; DG, degasser; P, pump; I, injector; $\mathrm{D}, \mathrm{CL}$ detector; ECR, electrochemical reactor; PMT, photomultiplier tube; DP, data processor. $X$ is the tube length between the mixing tee and the detection cell. The default value of $X$ is $4 \mathrm{~cm}$.

Table 1 Normalized CL intensity of selected monoheteroaromatic compounds

\begin{tabular}{lrrr}
\hline \multirow{1}{*}{ Compound } & \multicolumn{2}{c}{$\begin{array}{c}\text { Normalized CL } \\
\text { intensity }^{\mathrm{a}}\end{array}$} & \\
\cline { 2 - 3 } & $\mathrm{pH} 2.8$ & $\mathrm{pH} 7.1$ & \\
\cline { 2 - 3 } Pyrrole & 29 & 36 & 8.23 \\
2,5-Dimethylpyrrole & 1090 & 8 & 7.69 \\
Thiophene & 0 & -8 & 8.85 \\
2-Methylthiophene & 0 & -8 & 8.59 \\
2,5-Dimethylthiophene & 2950 & 476 & 8.1 \\
2,5-Thiophenedicarboxylic acid & 0 & 0 & - \\
2,5-Dimethylfuran & 99 & 16 & - \\
L-Tryptophan & 240 & 112 & 7.5 \\
3-(Thianaphthene-3-yl)-L-alanine & 686 & 3200 & - \\
Pyridine & -4 & -16 & 9.26 \\
2,6-Dimethylpyridine & -4 & -8 & 8.9 \\
Thianaphthene & 0 & 0 & 8.2 \\
Dibenzothiophene & 0 & 0 & 7.93 \\
\hline
\end{tabular}

Sample: $10 \mu \mathrm{M}$ each. Conditions are described in the text. a. Negative values show the decrease in the CL from the background CL.

ruthenium(II) chloride hexahydrate $\left(\mathrm{Ru}(\mathrm{bpy})_{3} \mathrm{Cl}_{2} \cdot 6 \mathrm{H}_{2} \mathrm{O}\right)$. $\mathrm{Ru}(\mathrm{bpy})_{3} \mathrm{Cl}_{2} \cdot 6 \mathrm{H}_{2} \mathrm{O}$ was prepared in our laboratory. Stock solutions of all heteroaromatic compounds were prepared with acetonitrile and/or water and were kept in the dark and cool. The stock solutions were diluted with the carrier before use. Water for all solutions was purified using a GS-200 automatic water distillation apparatus (Advantec, Tokyo, Japan) and then a Milli-QII water purification system (Millipore, Bedford, MA, USA).

\section{Apparatus and procedure}

Flow injection analysis (FIA) experiments were conducted with the CL detection system shown in Fig. 1A. A GL Science PU611 pump (GL Science, Tokyo, Japan) was used to deliver solutions; such solutions were purged with a Shodex Degas (Showa Denko, Tokyo, Japan). The carrier solution was delivered to a Comet 3000 chemiluminescence detector (Comet, Kawasaki, Japan) through a Reodyne 7125 sample injector (Rheodyne, Cotati, CA, USA) equipped with a $20 \mu$ injector loop. The $0.1 \mathrm{M}$ phosphate buffer solution, which was mixed with the carrier stream after the injector in order to control the $\mathrm{pH}$ of the chemiluminescent reaction, was delivered at a flow rate of $0.1 \mathrm{ml} \mathrm{min}{ }^{-1}$. A $\left.0.5 \mathrm{mM} \mathrm{Ru(bpy)}\right)_{3}{ }^{2+}$ solution was prepared by dissolving a weighed quantity of $\mathrm{Ru}(\mathrm{bpy})_{3} \mathrm{Cl}_{2} \cdot 6 \mathrm{H}_{2} \mathrm{O}$ in $0.1 \mathrm{M}$ sodium sulfate solution containing $1 \mathrm{mM}$ sulfuric acid.
The $\mathrm{Ru}(\mathrm{bpy})_{3^{2+}}$ solution was delivered at a flow rate of $0.3 \mathrm{ml}$ $\mathrm{min}^{-1}$ and oxidized to $\mathrm{Ru}(\mathrm{bpy})_{3}{ }^{3+}$ by the controlled-current electrolysis method (Galvanostat Comet 3000). The electrolytic current of the electrochemical reactor was set at $80 \mu \mathrm{A}$. The carrier and $\mathrm{Ru}(\mathrm{bpy})_{3}{ }^{3+}$ solutions were mixed and pumped continuously through the spiral flow cell in the detector. FIA profiles were recorded with a Hitachi D-2500 ChromatoIntegrator (Hitachi, Tokyo, Japan). UV-VIS detection was performed on a Hitachi L-4200 UV-VIS detector (Hitachi, Tokyo, Japan). All connecting PTFE tubing was $0.5 \mathrm{~mm}$ i.d..

\section{Results and Discussion}

Reaction of Ru(bpy) $)_{3}{ }^{3+}$ with various heteroaromatic compounds

$\mathrm{CL}$ intensities of $\mathrm{Ru}(\mathrm{bpy})_{3^{3+}}$ with various heteroaromatic compounds were measured; the 3-pump FIA system was used in order to control the $\mathrm{pH}$ of the chemiluminescent reaction. The carrier solutions were an acetonitrile/water $=7 / 3(\mathrm{v} / \mathrm{v})$ for mono-heteroaromatic compounds and an acetonitrile/water $=$ $2 / 8(\mathrm{v} / \mathrm{v})$ for di- and tri-heteroaromatic compounds. The carrier solutions were delivered at a flow rate of $0.8 \mathrm{ml} \mathrm{min}^{-1}$. Because $\mathrm{Ru}(\mathrm{bpy}) 3_{3}{ }^{3+}$ reacts with hydroxide and emits weak $\mathrm{CL},{ }^{19}$ the noise level rises with an increase in $\mathrm{pH}$ of the chemiluminescent reaction. In this study, we evaluated the CL intensity including the noise level defined as normalized CL intensity (signal/noise).

Normalized CL intensities observed for mono-heteroaromatic compounds are shown in Table 1. At acidic conditions, strong CL signals were observed for $\alpha$-position-dialkylated fivemembered heteroaromatic compounds, such as DMT, DMP, and DMF, whereas no CL was observed for 2,6-dimethylpyridine which is an $\alpha$-position-dialkylated six-membered heteroaromatic compound. This result may be attributed to a difference in the $\pi$-electron density of the aromatic rings, because it is known that five-membered mono-heteroaromatic compounds, such as pyrrole, have higher $\pi$-electron density than the corresponding six-membered mono-heteroaromatic compounds, such as pyridine, and have higher reactivity to electrophilic reactions compared with benzene derivatives. In addition, DMT, which has two electron-donating methyl groups, produced strong CL, whereas thiophene, 2-methylthiophene, and 2,5-thiophenedicarboxylic acid, which have no electronwithdrawing/donating groups, one electron-donating methyl group, and two electron-withdrawing carboxylic groups, respectively, did not produce CL. This result also suggests that generation of $\mathrm{CL}$ upon the reaction of $\mathrm{Ru}(\mathrm{bpy})_{3}{ }^{3+}$ with heteroaromatic compounds is dependent on the $\pi$-electron density of the aromatic ring. The following facts may support 
Table 2 Normalized CL intensity of selected di- and triheteroaromatic compounds

\begin{tabular}{lrrr}
\hline \multirow{2}{*}{ Compound } & \multicolumn{3}{c}{ Normalized CL intensity } \\
\cline { 2 - 4 } & $\mathrm{pH} 2.4$ & $\mathrm{pH} 7.1$ & $\mathrm{pH} 9.4$ \\
\hline Imidazole & 36 & 53 & 188 \\
Pyrazole & 40 & 42 & 16 \\
Thiazole & 24 & 14 & 6 \\
$N$-Methylimidazole & 24 & 34 & 18 \\
2-Ethylimidazole & 24 & 34 & 80 \\
4-Methylimidazole & 16 & 114 & 200 \\
L-Histidine & 0 & 3250 & 628 \\
Histamine & 8 & 168 & 64 \\
Cimetidine & 16 & 117 & 1030 \\
$\beta$-Alanyl-L-histidine & 16 & 120 & 196 \\
4-Hydroxymethyl-5-methylimidazole & 0 & 68 & 120 \\
1,2,4-Triazole & 20 & 12 & 0 \\
3-Amino-1,2,4-triazole (Amitrole) & 0 & 242 & 24 \\
\hline
\end{tabular}

Sample: $10 \mu \mathrm{M}$ each. Conditions are described in the text.

this conclusion. The generation of CL upon the reaction of $\mathrm{Ru}$ (bpy) ${ }_{3}{ }^{3+}$ with aliphatic conjugated dienes was dependent on $\pi$-electron density; ${ }^{17}$ no CL was observed for the reaction of $\mathrm{Ru}(\mathrm{bpy})_{3}{ }^{3+}$ with benzene derivatives having various functional groups without photochemical oxidation. ${ }^{20}$

Noffsinger and Danielson indicated that the ionization potential (I.P.) is a useful indicator for finding additional reducing agents for the $\mathrm{Ru}(\mathrm{bpy})_{3}{ }^{3+} \mathrm{CL}$ reaction. ${ }^{7}$ Chen also reported that the $\mathrm{CL}$ intensity of alkaloids containing a tertiary amine moiety increases in reverse order of I.P. values. ${ }^{8}$ Table 1 lists the I.P. values as measured by UV photoelectron spectroscopy or electron impact for each compound as reported in the literature. ${ }^{21}$ The mono-heteroaromatic compounds that produced significant CL intensities have the I.P. values ranging from 7.5 to $8.2 \mathrm{eV}$. Such I.P. values are close to those of aliphatic tertiary amines, ${ }^{7}$ which are the most suitable reducing agents for the chemiluminescent reaction of $\mathrm{Ru}(\mathrm{bpy})_{3}{ }^{3+}$. Although I.P. values for dibenzothiophene and thianaphthene fall in the range of energies for the chemiluminescent monoheteroaromatic compounds $(7.5-8.2 \mathrm{eV})$, they did not produce CL. Similar results have been reported for amines. ${ }^{7}$ For example, although triphenylamine, diphenylamine, and aniline have the I.P. values ranging from 7 to $8 \mathrm{eV}$, they do not produce CL. Knight and Greenway have explained the reason as follows. When the phenyl group is directly attached to the nitrogen, the generated radical intermediates are excessively stabilized by the resonance stabilization. They will be less reactive towards the $\mathrm{Ru}(\mathrm{bpy})_{3^{3+}}$ and hence hinder the $\mathrm{CL}$ intensity. ${ }^{22}$ As dibenzothiophene and thianaphthene are heteroaromatic compounds that have thiophene and benzene rings fused together, we assume that the reason why these compounds do not produce CL is the same as that for aromatic amines. The CL intensities increased in the order DMF $<$ DMP $<$ DMT, even though the I.P. values are DMP $<$ DMT (Table 1). This order of CL intensity is the same as the order of aromaticity. This may be because the stability of aromatic rings is involved in the character of the generated radical intermediate.

Normalized CL intensities observed for five-membered diand tri-heteroaromatic compounds are shown in Table 2. The generation of CL was observed for all compounds examined, although the $\mathrm{CL}$ intensities of these compounds were rather weak compared with those of $\alpha$-position-dialkylated five- membered mono-heteroaromatic compounds. Most of the compounds examined here produced more intense CL at neutral or basic conditions rather than acidic conditions. As these compounds have at least one nitrogen atom having a lone pair of electrons, the increased CL intensity at neutral or basic conditions would be involved in the lone pair of electrons released from a proton (imidazole, $\mathrm{p} K_{\mathrm{a}}$ 7.0; pyrazole, $\mathrm{p} K_{\mathrm{a}} 2.5$ ). However, pyridine $\left(\mathrm{p} K_{\mathrm{a}} 5.4\right)$ that has a nitrogen atom possessing a lone pair of electrons did not produce $\mathrm{CL}$ even at $\mathrm{pH} 7.1$ (Table 1) because of its low $\pi$-electron density. The CL intensity of 3-amino-1,2,4-triazole was nearly twenty times more than that of 1,2,4-triazole at $\mathrm{pH} 7.1$ (Table 2). The amino group of 3-amino-1,2,4-triazole is electron-donating and therefore is a very potent activator to the aromatic ring. These results suggest that the $\pi$-electron density of aromatic rings would be an important factor for the chemiluminescent reaction of $\mathrm{Ru}(\mathrm{bpy}) 3^{3+}$ with heteroaromatic compounds.

Previous reports have shown that histidine ${ }^{23}$ and tryptophan ${ }^{24}$ are more reactive amino acids for the chemiluminescent reaction of $\mathrm{Ru}(\mathrm{bpy})_{3}{ }^{3+}$. In our research as well, amino acids having a heteroaromatic ring (tryptophan, 3-(thianaphthen-3yl)-L-alanine, and histidine) gave strong CL, as shown in Tables 1 and 2. The strong CL of these amino acids is thought to be caused by not only the chemiluminescent reaction of an amine moiety but also the chemiluminescent reaction of a fivemembered heteroaromatic ring. However, the CL intensity of histidine was stronger by thirty times than that of 4methylimidazole at $\mathrm{pH} 7.1$ (Table 2) even though both histidine and 4-methylimidazole have a five-membered aromatic ring. In order to clarify the reason for this, we also examined the chemiluminescent reactions of $\operatorname{Ru}(\mathrm{bpy})_{3}{ }^{3+}$ with histamine and $\beta$ alanyl-L-histidine. Histamine has the structure with no carboxylic group of histidine and $\beta$-alanyl-L-histidine has the structure in which the amino group of histidine forms an amide bond. The CL intensities of these compounds were as much as that of 4-methylimidazole. These results suggest that the strong $\mathrm{CL}$ of histidine results from the presence of both amino acid and imidazole moieties in the molecule. Previously, Uchikura and Kirisawa have reported on the chemiluminescent reaction of $\mathrm{Ru}(\mathrm{bpy}) 3_{3}{ }^{3+}$ with indole derivatives such as tryptophan and 3indoleacetic acid. ${ }^{24}$ They have indicated that the CL intensity of tryptophan is about ten-times stronger than that of other indole derivatives. We have examined the chemiluminescent reaction of $\mathrm{Ru}(\mathrm{bpy})_{3^{3+}}$ with thianaphthene and 3-(thianaphthen-3-yl)-Lalanine as analogues of indole and tryptophan, respectively. The strong CL intensity was observed for 3-(thianaphthen-3-yl)L-alanine, whereas thianaphthene did not produce CL, as shown in Table 1. These results suggest that a five-membered heteroaromatic ring increases the chemiluminescent activity of amino acids rather than the synergistic effect of both the chemiluminescent reaction of an amine moiety and the chemiluminescent reaction of a heteroaromatic ring for the strong CL of amino acids containing a five-membered heteroaromatic ring.

\section{Characterization of $R u(b p y) 3_{3}^{3+} /$ heteroaromatic compound chemiluminescent reaction}

DMT, DMP, and DMF were considered for this study. The effect of the $\mathrm{pH}$ on the $\mathrm{CL}$ intensity was examined with the aid of the 3-pump FIA system. The carrier was an acetonitrile/water $=5 / 5(\mathrm{v} / \mathrm{v})$ mixture delivered at a flow rate of $0.5 \mathrm{ml} / \mathrm{min}$. Other conditions are the same as those described in Apparatus and procedure. Figure 2 shows the obtained results. The signal-to-noise ratio was plotted as a function of the $\mathrm{pH}$ at which the chemiluminescent reaction was conducted. DMT, 


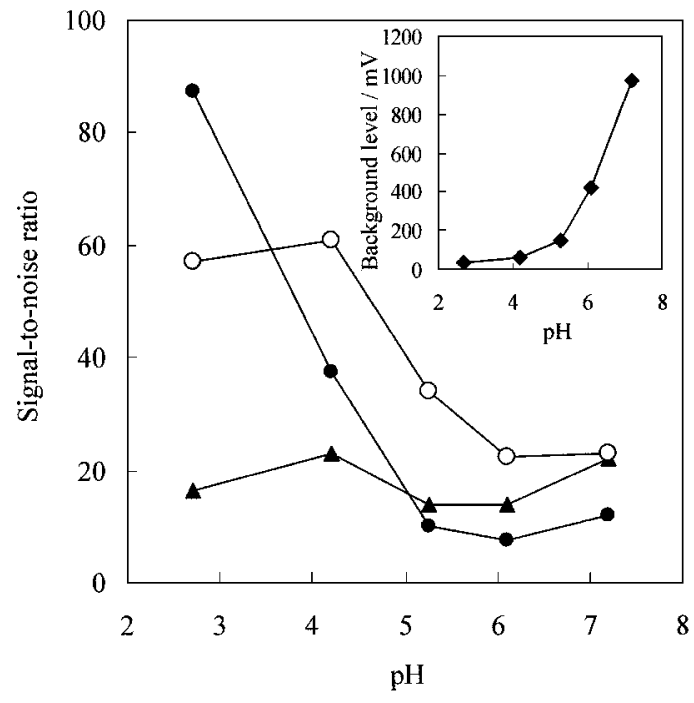

Fig. 2 Effect of the $\mathrm{pH}$ on the CL intensity for DMT, DMP, and DMF obtained by the 3-pump FIA system. Sample: $1 \mu$ M DMT (O), $5 \mu \mathrm{M}$ DMP (•), and $10 \mu \mathrm{M} \operatorname{DMF}(\boldsymbol{\Lambda})$. Inset: background level as a function of $\mathrm{pH}$.

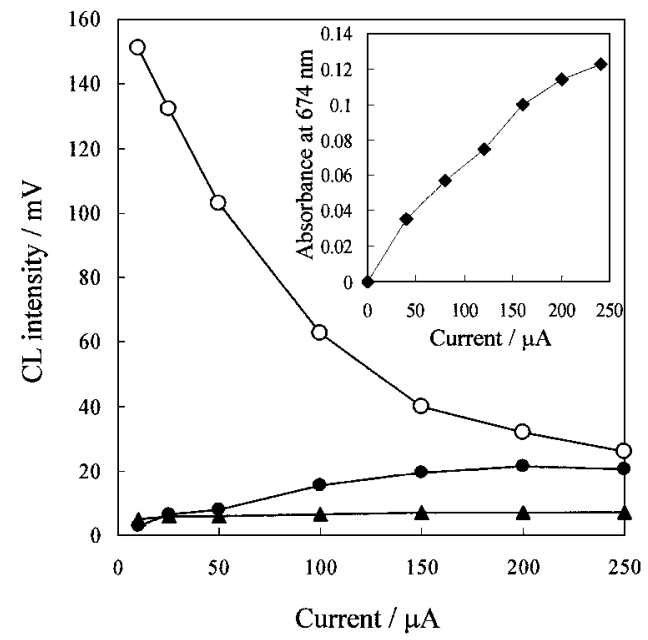

Fig. 3 Effect of the electrolytic current of ECR on the CL intensity for DMT, DMP, and DMF obtained by the 2-pump FIA system. Sample: $4 \mu \mathrm{M}$ DMT (O), $4 \mu \mathrm{M}$ DMP $(\bullet)$, and $4 \mu \mathrm{M}$ DMF (4). Inset: absorbance at $674 \mathrm{~nm}$ as a function of the electrolytic current.

DMP, and DMF showed a maximum signal-to-noise ratio under acidic conditions. At acidic conditions, the CL intensities of most of the previously reported reducing agents, such as amines $^{7}$ and amino acids, ${ }^{23}$ are very weak. Therefore, the detection of five-membered mono-heteroaromatic compounds at lower $\mathrm{pH}$ should become a more selective analytical method.

The effect of the electrolytic current on the CL intensity was examined with the aid of the 2-pump FIA system in which $\mathrm{P}_{3}$ and $\mathrm{B}$ represented in Fig. 1A were removed. The carrier was an acetonitrile/water $=5 / 5(\mathrm{v} / \mathrm{v})$ mixture delivered at a flow rate of $0.5 \mathrm{ml} / \mathrm{min}$. A $0.5 \mathrm{mM} \mathrm{Ru}(\mathrm{bpy})_{3}{ }^{2+}$ solution was prepared with a $10 \mathrm{mM}$ sulfuric acid solution and delivered at a flow rate of 0.3 $\mathrm{ml} \mathrm{min}{ }^{-1}$. The electrolytic current of the ECR was varied. When the $\mathrm{Ru}(\mathrm{bpy})_{3}{ }^{2+}$ solution was used, the $\mathrm{pH}$ value of the chemiluminescent reaction was near 2 . In order to evaluate the relationship between the electrolytic current and the generated

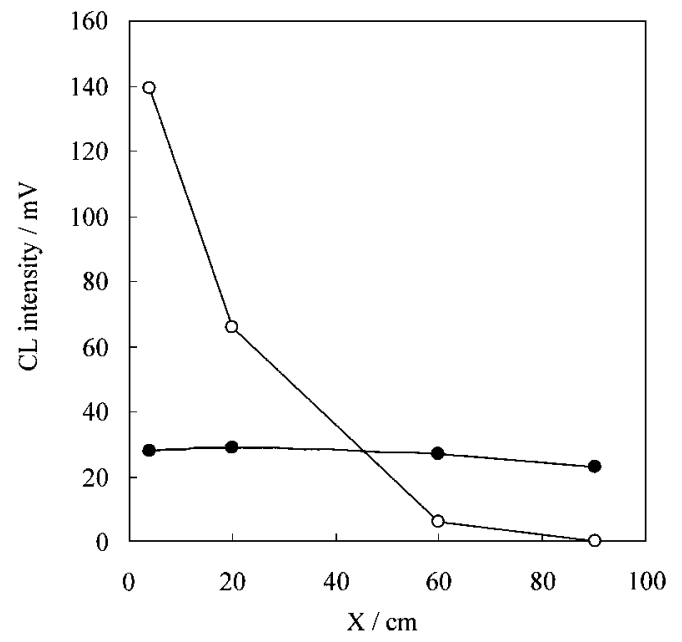

Fig. 4 Effect of the tube length on the CL intensity for DMT and DMP obtained by the 2-pump FIA system. Sample: $10 \mu \mathrm{M}$ DMT (O) and $10 \mu \mathrm{M} \operatorname{DMP}(\bullet)$

amount of $\mathrm{Ru}(\mathrm{bpy}) 3^{3+}$, an UV-VIS detector was attached between the ECR and the mixing tee as represented in Fig. 1B. Since $\mathrm{Ru}(\mathrm{bpy})_{3}{ }^{3+}$ has specific absorption at $674 \mathrm{~nm},{ }^{25}$ the UVVIS detector was set at this wavelength. Figure 3 shows the obtained results. The result obtained for DMT is especially characteristic. In general, the CL intensity increases with increasing the electrolytic current ${ }^{10,18}$ due to an increase in the amount of $\mathrm{Ru}(\mathrm{bpy})_{3}{ }^{3+}$. In fact, it was confirmed that the amount of $\mathrm{Ru}(\mathrm{bpy})_{3^{3+}}$ increased with increasing the electrolytic current and then the CL intensities of DMP and DMF increased as shown in Fig. 3. However, the CL intensity of DMT increased with decreasing the electrolytic current down to at least $1 \mu \mathrm{A}$. We think the reason for this decrease is that the chemiluminescent reaction of $\mathrm{Ru}(\mathrm{bpy})_{3^{3+}}$ with DMT is almost complete before reaching the detection cell in the presence of a large amount of $\mathrm{Ru}(\mathrm{bpy})_{3}{ }^{3+}$, because the sample solution is mixed with the $\mathrm{Ru}(\mathrm{bpy})_{3}{ }^{3+}$ solution before the detection cell (Fig. 1A).

In order to confirm this assumption, we controlled the time from mixing the sample solution with the $\mathrm{Ru}(\mathrm{bpy})_{3^{3+}}$ solution until reaching the detection cell by changing the length of the tube that is represented as $X$ in Fig. 1B. The electrolytic current of the ECR was set at $80 \mu \mathrm{A}$. Other experimental conditions were same as described above. As shown in Fig. 4, the CL intensity of DMT greatly decreased with increasing the length of the tube, whereas the CL intensity of DMP was almost constant. These results suggest that the rate of chemiluminescent reaction of $\mathrm{Ru}(\mathrm{bpy})_{3}{ }^{3+}$ with DMT is very fast and so the $\mathrm{CL}$ of this reaction is not observed effectively in our detection system.

\section{Conclusions}

Novel reducing agents for the chemiluminescent reaction of $\mathrm{Ru}(\mathrm{bpy}) 3^{3+}$ have been found. The relationship between the structural attributes of various heteroaromatic compounds and their activity for the chemiluminescent reaction of $\mathrm{Ru}(\mathrm{bpy})_{3}{ }^{3+}$ was examined. Above all, thiophene derivatives were effective reducing agents for the chemiluminescent reaction of $\mathrm{Ru}(\mathrm{bpy})_{3}{ }^{3+}$. Since DMT showed high sensitivity at the acidic conditions and the lower electrolytic current, development of 
derivatization reagents having a DMT moiety will be one of the successful applications of this chemiluminescent reaction. We have already developed (2,4,5-trimethylthiophene-3-yl)acetic acid as a precolumn derivatization reagent. The applications of this reagent to HPLC determination of alkylphenols and polyols are under consideration. In addtion, we are investigating further details of the reaction of $\mathrm{Ru}(\mathrm{bpy})_{3}{ }^{3+}$ with thiophene derivatives

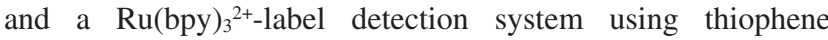
derivatives as coreactants. The reason why heteroaromatic rings increase the chemiluminescent activity of an amino acid moiety is also under consideration.

\section{Acknowledgements}

The present work was partially supported by a Grant-in-Aid for Scientific Research (No. 18550073) from the Ministry of Education, Culture, Sports, Science and Technology, Japan.

\section{References}

1. W.-Y. Lee, Mikrochim. Acta, 1997, 127, 19.

2. R. D. Gerardi, N. W. Barnett, and S. W. Lewis, Anal. Chim. Acta, 1999, 378, 1 .

3. M. M. Richter, Chem. Rev., 2004, 104, 3003.

4. X.-B. Yin and E. Wang, Anal. Chim. Acta, 2005, 533, 113.

5. B. A. Gorman, P. S. Francis, and N. W. Barnett, Analyst, 2006, 131, 616.

6. A. J. Bard, "Electrogenerated Chemiluminescence", 2004, Marcel Dekker, New York.

7. J. B. Noffsinger and N. D. Danielson, Anal. Chem., 1987, 59,865 .

8. X. Chen, C. Yi, M. Li, X. Lu, Z. Li, P. Li, and X. Wang, Anal. Chim. Acta, 2002, 466, 79.
9. H. N. Choi, S.-H. Cho, Y.-J. Park, D. W. Lee, and W.-Y. Lee, Anal. Chim. Acta, 2005, 541, 47.

10. H. Kodamatani, K. Saito, N. Niina, S. Yamazaki, and Y. Tanaka, J. Chromatogr. A, 2005, 1100, 26.

11. N. W. Barnett, B. J. Hindson, and S. W. Lewis, Anal. Chim. Acta, 1999, 384, 151.

12. J. W. Costin, N. W. Barnett, and S. W. Lewis, Talanta, 2004, 64, 894.

13. X.-B. Yin, J. Kang, L. Fang, X. Yang, and E. Wang, J. Chromatogr. A, 2004, 1055, 223.

14. L. Fang, X.-B. Yin, X. Sun, and E. Wang, Anal. Chim. Acta, 2005, 537, 25.

15. X.-B. Yin, Y. Du, X. Yang, and E. Wang, J. Chromatogr. A, 2005, 1091, 158.

16. D. R. Deaver, Nature, $\mathbf{1 9 9 5}, 377,758$

17. H. Kodamatani, K. Saito, A. Muromatsu, N. Niina, and S. Yamazaki, Anal. Lett., 2005, 38, 291.

18. H. Kodamatani, K. Saito, N. Niina, and S. Yamazaki, J. Ion Exchange, 2003, 14(Supplement), 229.

19. D. M. Hercules and F. E. Lytle, J. Am. Chem. Soc., 1966, 88,4745 .

20. H. Kodamatani, H. Shimizu, K. Saito, S. Yamazaki, and Y. Tanaka, J. Chromatogr. A, 2006, 1102, 200.

21. D. L. Rhoda and G. L. Sharon, "Ionization Potential and Appearance Potential Measurements", 1971 -1981, NSRDS-NBS 71, Ion Kinetics and Energetics Data Center, National Measurement Laboratory, National Bureau of Standards, Washington, D.C.

22. A. W. Knight and G. M. Greenway, Analyst, 1996, 121, $101 \mathrm{R}$.

23. L. He, K. A. Cox, and N. D. Danielson, Anal. Lett., 1990, 23, 195 .

24. K. Uchikura and M. Kirisawa, Chem. Lett., 1991, 1373.

25. R. D. Gerardi, N. W. Barnett, and P. Jones, Anal. Chim. Acta, 1999, 388, 1 . 